\title{
Ningen Dock: Japan's unique comprehensive health checkup system for early detection of disease
}

\author{
Jun Lu* \\ Medical Examination Center, National Center for Global Health and Medicine, Tokyo, Japan.
}

\begin{abstract}
Japan's comprehensive health checkup - Ningen Dock - is a unique system for early detection of disease that has developed over the years along with the country's universal health insurance system. Ningen Dock is currently offered at 1,727 facilities nationwide, involving about 3.7 million people annually. The development of the comprehensive health checkup system may be one reason for Japan's long life expectancy. The major purpose of the comprehensive health checkup system is to maintain health in three main ways: early detection of cancer, detection of lifestyle-related diseases, and confirmation of health status. Here, the history and current status of Ningen Dock in Japan, tests and examinations included in the comprehensive health checkup system, the effectiveness of those checkups, and their advantages and disadvantages are described.
\end{abstract}

Keywords: Ningen Dock, comprehensive health checkups, Japan

\section{Introduction}

Japan's comprehensive health checkup - Ningen Dock - is a unique system for early detection of disease that has developed over the years along with the country's universal health insurance system. "Ningen" means human in Japanese, "Dock" is derived from the dockyard for inspecting and repairing ships. "Ningen Dock" is similar to the medical checkup system in which people visit a facility for a medical checkup. As a comprehensive health checkup system, Ningen Dock have advanced in Japan and are currently offered at 1,727 facilities nationwide, involving about 3.7 million people annually (1).

The development of the comprehensive health checkup system may be one reason for Japan's long life expectancy (2). The major purpose of the comprehensive health checkup system is to maintain health in three main ways: early detection of cancer, detection of lifestyle-related diseases, and confirmation of health status. Although the cost of such an examination is borne by the examinee, this system will help to reduce the country's overall health care expenditures through early detection and early treatment.

Symptom-based medical care is being major developed around the world, so there is limited public awareness of the comprehensive health checkup system. Facilities perform comprehensive health checkups in countries such as China (3), Thailand (4), Singapore (5), and Vietnam (6). Here, the history and current status of
Ningen Dock in Japan, tests and examinations included in the comprehensive health checkup system, the effectiveness of those checkups, and their advantages and disadvantages are described.

\section{History of Ningen Dock}

In Japan, July 12th is "Ningen Dock Day". The first systematic Ningen Dock was created at the National Tokyo Daiichi Hospital - now the National Center for Global Health and Medicine (NCGM) - on July 12, 1954. At the time, the examination was called "a detailed physical examination involving short-term hospitalization", and a full-scale medical examination was conducted over 6 days. This is the origin of comprehensive medical examinations in Japan (7).

In 1955, an outpatient health checkup over five days, mainly involving internal medicine, was instituted at the Aichi Prefectural Central Health Counseling Center. On 1958, a health checkup involving short-term hospitalization for one night and two days was devised at St. Luke's Hospital, and it widely served as the basic format for a health checkup (8). This multi-day health checkup was undergone by about 300,000 people annually in 1994. Checkups have taken less and less time, and one-day health checkups are now possible. Today, one-day health checkups are the mainstream, and one-day and multi-day health checkups are undergone by more than 3.7 million people annually (Figure 1). 


\section{Current status of Ningen Dock}

Currently, Japanese medical examinations are roughly divided into legally mandated medical examinations and voluntary medical examinations (Table 1). Legally mandated medical examinations include medical examinations of local residents conducted by local governments, regular medical examinations conducted by companies and employee organizations, tuberculosis and lung cancer examinations, and health examinations based on the Maternal and Child Health Act and the School Health Act. Health checkups for the elderly have been conducted since 2008 for all participants in public medical insurance ages 40 to 74 (9).

In addition, voluntary medical examinations such as Ningen Dock are also conducted. Within the voluntary medical examination market in FY2016, "general health checkups" represented a market of 420 billion yen while "specialized health checkups" such as breast cancer screening and brain examinations represented a market of 45 billion yen. Over the past few years, the market for general health checkups has changed little, amounting to 484 billion yen in 2013, 464 billion yen in 2014, and 464 billion yen in 2015 (average 471 billion yen) $(10,11)$.

Tests and examinations that are part of a comprehensive health checkup

Each facility has some basic tests and examinations and optional tests and examinations, although there are some differences.

$$
3,000,000
$$

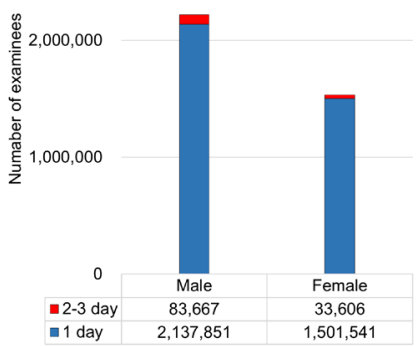

Figure 1. Average number of people who undergo Ningen Dock health checkup in Japan. (Apr. 2017-Mar. 2019). Source: Reference (1) 2017/2018 Report on a survey of member facilities (2021/3/31), with modifications.

\section{Basic tests and examinations}

Basic tests and examinations include an upper gastrointestinal tract test, physical measurements, medical examination, physiological function test, urine test, stool test, X-ray examination, abdominal ultrasound, blood test (general blood test, liver function test, renal function test, lipid metabolism test, pancreatic function test, sugar metabolism, test for gout, serology, thyroid test, test for tumor markers, and an immunological test), gynecological examination, etc. (12) (Table 2).

\section{Optional tests and examinations}

Various examinations and tests are conducted by each facility, including a lower gastrointestinal tract examination, breast examination, test for arteriosclerosis, brain examination, heart examination, pancreas examination, liver examination, bone examination, test for lifestyle-related diseases, test for Helicobacter pylori infection, test for human papillomavirus infection, PETCT scan, etc.

\section{Ningen Dock at the NCGM}

To provide tailored comprehensive health checkups suitable for everyone, the NCGM is striving to offer options to examinees beyond the basic tests and examinations (Figure 2).

Since 2010, encouraging medical tourism has been a key policy of the Japanese Government. The number of facilities catering to medical tourism has increased. Given that context, the current authors' facility - Ningen Dock at the NCGM - has endeavored to become an international medical facility since 2016. Various efforts have been made to enhance the facility's ability to cater to foreign examinees by translating explanations, test and examination guides, and consent forms, by providing guidance before tests and examinations and on-site interpretation for tests and examinations, and by publishing reports on the number of examinees and their country of origin. As a result of these efforts, the total number of foreign examinees as of March 2017 had increased about 10 -fold compared to the previous year. The number

Table 1. Comparison of legally mandated and voluntary medical examinations conducted annually in Japan

\begin{tabular}{|c|c|c|}
\hline & Legally mandated medical examinations & $\begin{array}{l}\text { Voluntary medical examination } \\
\text { (Comprehensive health checkup) }\end{array}$ \\
\hline Purpose & Maintain public health & Maintain personal health \\
\hline Contents & $\begin{array}{l}\text { Anthropometry, eyesight, hearing, blood pressure, chest X-ray, blood } \\
\text { test (hemoglobin, red blood cell count, ALT, AST, } \gamma \text {-GTP, triglycerides, } \\
\text { HDL-C, LDL-C, fasting blood glucose, HbA1c), urinalysis (protein, } \\
\text { sugar), electrocardiogram, doctor's consultation, etc. }\end{array}$ & $\begin{array}{l}\text { Includes the same tests/examinations } \\
\text { as the legally mandated medical } \\
\text { examination }\end{array}$ \\
\hline Number of examinees & About $28,000,000$ & About $3,700,000$ \\
\hline Market size & About 445 billion yen & About 471 billion yen \\
\hline
\end{tabular}


Table 2. Tests and examinations that are part of Ningen Dock health checkups

\begin{tabular}{|c|c|}
\hline Test/examination & Details \\
\hline Upper Gastrointestinal exam & Gastric camera (gastroscopy) or barium meal (stomach X-ray) \\
\hline Physical measurements & Height, weight, obesity index (BMI), body fat percentage, abdominal circumference \\
\hline General consultation & History taking, auscultation and percussion of the heart and lungs, palpation \\
\hline Physiological function test & $\begin{array}{l}\text { Blood pressure, electrocardiogram (at rest), hearing test (simple), visual acuity, intraocular pressure, ocular } \\
\left.\text { fundus, lung function test ( } \mathrm{FVC}, \mathrm{FVC} \%, \mathrm{FEV}_{1} \%\right)\end{array}$ \\
\hline \multicolumn{2}{|r|}{ 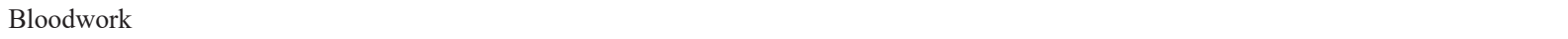 } \\
\hline General & $\begin{array}{l}\text { White blood cell count (differential count of leucocyte), red blood cell count, hemoglobin level, hematocrit, } \\
\text { platelet count, mean corpuscular volume (MCV), mean corpuscular hemoglobin }(\mathrm{MCH}) \text {, mean corpuscular } \\
\text { hemoglobin concentration (MCHC) }\end{array}$ \\
\hline Liver function & Total protein, albumin, total bilirubin, AST, ALT, $\gamma$-GTP, ALP \\
\hline Kidney function & Urea nitrogen, creatinine, electrolytes $(\mathrm{Na}, \mathrm{K}, \mathrm{Cl}, \mathrm{Ca}, \mathrm{P}, \mathrm{Mg})$ \\
\hline Lipid metabolism & Total cholesterol, HDL cholesterol, LDL cholesterol, triglycerides \\
\hline Pancreatic function & Amylase \\
\hline Glycometabolism & Fasting blood glucose, glycohemoglobin (HbA1c) \\
\hline Gout & Uric acid \\
\hline Serological tests & $\begin{array}{l}\text { Hs-CRP, syphilis reaction, hepatitis virus test (HBs antigen and antibody, HCV antibody), blood type (ABO, } \\
\text { Rh type) }\end{array}$ \\
\hline Thyroid gland & TSH, FT4 \\
\hline Tumor marker & PSA (male only), CA125 (female only), CEA \\
\hline Rheumatoid & RF (Rheumatoid factor) \\
\hline Urine analysis & Urine specific gravity, $\mathrm{pH}$, protein, glucose, occult blood, sediment, urine microalbumin \\
\hline Stool analysis & Occult blood reaction (samples to be collected on 2 separate times) \\
\hline X-ray examination & Chest X-ray (2 directions) \\
\hline Abdominal ultrasonography & Liver, gallbladder, pancreas, kidneys, spleen, and other organs \\
\hline Gynecology (female only) & Pelvic exam, speculum exam, transvaginal ultrasonography, Pap smear (cervical screening) \\
\hline
\end{tabular}

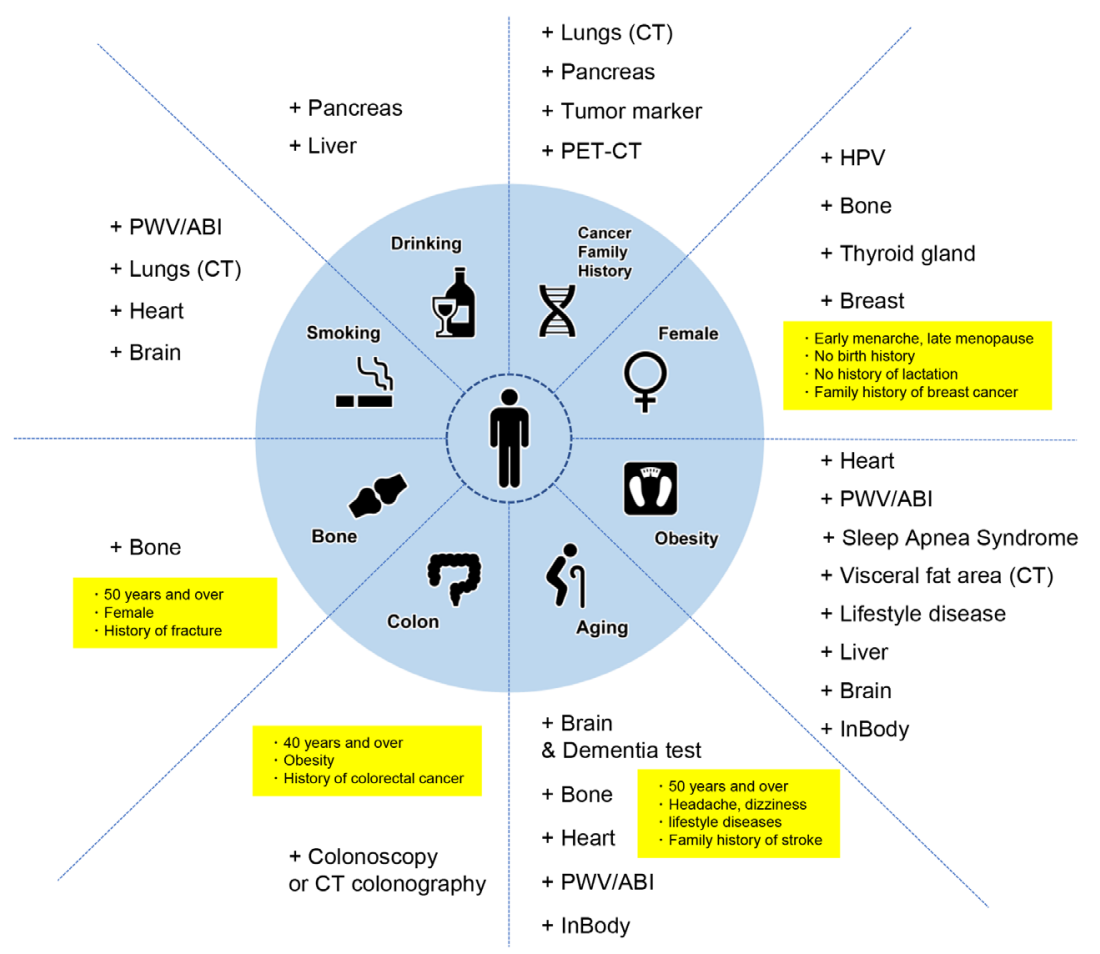

Figure 2. Ningen Dock: Rubric for selecting optional tests and examinations to undergo as part of a personalized medical examination. As an example, the general condition and risk of a 45 -year-old smoker could be evaluated by adding a colonoscopy, brain examination, heart examination, lung examination, and test for arteriosclerosis. The idea is to be able to perform a tailored examination that is better suited to the individual.

of Vietnamese examines increased rapidly after September 2017. Prior to 2019, the number of foreign examinees totaled 3,385 , accounting for $28 \%$ of all examinees, and foreign examinees accounted for more than $50 \%$ of the facility's total revenue (Figure 3).

\section{Effectiveness, advantages, and disadvantages of Ningen Dock}

Two year of data from the latest survey on the five major cancers by the Japan Society of Ningen Dock 


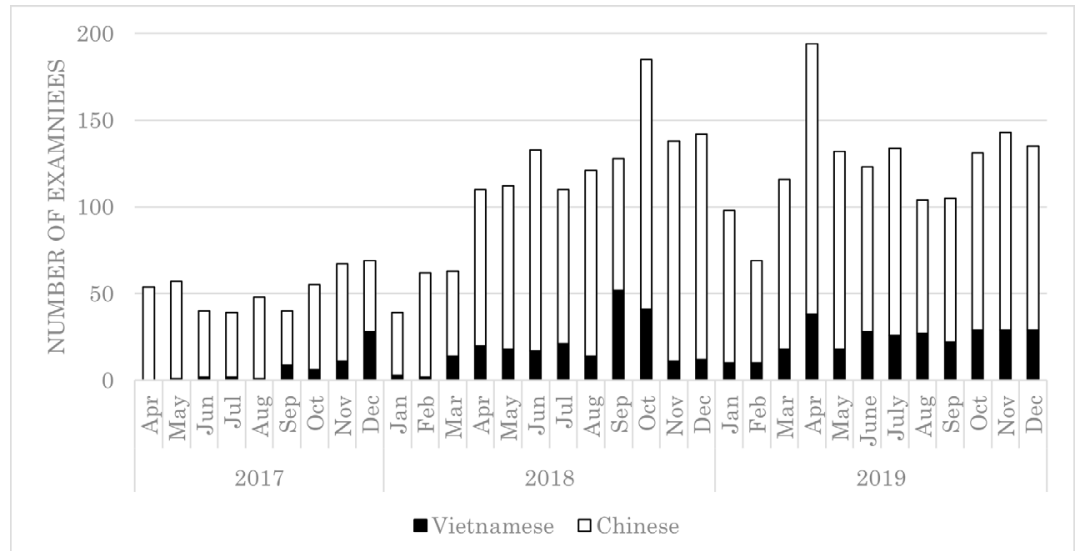

Figure 3. Changes in the number of foreigners visiting the Ningen Dock at the NCGM.

Table 3. Number of cancers found in individuals undergoing Ningen Dock health checkups (Apr. 2016 Mar. 2018)

\begin{tabular}{lccc}
\hline Examination & Number of examinees & Number of cancers found (including suspected) & Cancer confirmed \\
\hline Lung cancer & $6,395,860$ & 26,367 & 1,754 \\
Gastric cancer & $5,681,805$ & 39,828 & 4,526 \\
Colorectal cancer & $6,107,352$ & 83,587 & 4,239 \\
Breast cancer & $2,055,693$ & 27,490 & 4,463 \\
Cervical cancer & $1,772,270$ & 9,746 & 772 \\
\hline
\end{tabular}

Data source: Reference (1) 2017/2018 Report on a survey of member facilities (2021/3/31), with modifications.

indicated that the rate of cancer detection, including suspected lung cancer, in comprehensive health checkups was approximately $0.41 \%$. In $6.65 \%$ of examines, lung cancer was definitively diagnosed. The rate of detection of gastric cancer was $0.7 \%$, and gastric cancer was confirmed in $11.36 \%$ of examines. The rate of detection of breast cancer is the highest, with a rate of detection of $1.34 \%$, and cancer was confirmed in $16.23 \%$ of examinees (Table 3 ).

The biggest advantage of comprehensive health checkups is preserving life through early detection and early treatment. Since there is a high possibility that the patient can be cured at an early stage and the treatment is often less burdensome, the patient generally has less of a physical, financial, or temporal burden. Regular medical examinations are required so that disease can be found at an early stage.

A disadvantage of comprehensive health checkups is that the determination/results of the medical examination are not $100 \%$ correct. Although testing and examination accuracy and technology have made remarkable progress, there are still some situations in which disease may be overlooked. A comprehensive health checkup can also result in a "false positive" (13) and, as a result, "overdiagnosis" leading to unnecessary testing and treatment. Unnecessary tests and examinations may strain the body physically (pain, radiation exposure, etc.) and psychologically. One should undergo a comprehensive health checkup after considering its advantages and disadvantages.

\section{Conclusion}

There are still various aspects of the global problem of aging that still need to be addressed, such as early detection and treatment of disease and tests and examinations suited to the individual, but Japan's unique comprehensive health checkup system - Ningen Dock - should help in that regard. Even though the cost of such tests and examinations is born by individual examines themselves, this approach should help to reduce national health care costs through early detection and treatment.

\section{Funding: None.}

Conflict of Interest: The author has no conflicts of interest to disclose.

\section{References}

1. Committee to Survey Facilities Performing Comprehensive Health Checkups. 2017/2018 Report on a survey of member facilities (2021/3/31). https://www. ningen-dock.jp/wp/wp-content/uploads/2013/09/d36e0ce b105a39e3b9a9b519b10affd1-4.pdf (accessed August 5, 2021). (in Japanese)

2. Ministry of Health, Labor, and Welfare, Overview of the 2020 simplified life table https://www.mhlw.go.jp/toukei/ saikin/hw/life/life20/dl/life18-15.pdf (accessed August 5, 2021). (in Japanese)

3. Shanghai Senmao Clinic. https://senmaoclinic.co.jp/ 
(accessed October 15, 2021). (in Japanese)

4. Samitive Hospital. https://www.samitivejhospitals.com/ (accessed October 15, 2021)

5. Raffle Japanese Clinic. https://rafflesj-clinic.com/ (accessed October 15, 2021). (in Japanese)

6. Cho Ray Hospital. https://bvcrheci.vn/en/ (accessed October 15, 2021)

7. Japan Society of Ningen Dock. July 12th is Ningen Dock Day. https://www.ningen-dock.jp/0712dock (accessed August 5, 2021). (in Japanese)

8. Iwatsuka T. History and current status of comprehensive medical examinations in Japan. Jpns. J Multiphasic Health Testing and Service. 1994; 21:370-376. (in Japanese)

9. E-Healthnet. Health information site for prevention of lifestyle-related diseases. https://www.e-healthnet.mhlw. go.jp/information/others/metabolic.html (accessed August 5, 2021). (in Japanese)

10. Yano Research Institute. Conduct of a survey on the medical checkup and Ningen Dock market (2019). https://www.yano.co.jp/press-release/show/press_id/2300 (accessed August 5, 2021). (in Japanese)

11. Yano Research Institute. Conduct of a survey on the medical checkup and Ningen Dock market (2016). https://www.yano.co.jp/press-release/show/press_id/1542 (accessed August 5, 2021). (in Japanese)

12. National Center for Global Health and Medicine, Medical Examination Center. http://www.hosp.ncgm.go.jp/cmc/040/ menu.html (accessed August 5, 2021) (in Japanese)

13. Japan Cancer Society. Promotion of cancer prevention and cancer screening. https://www.jcancer.jp/about_cancer_ and_checkup (accessed August 5, 2021). (in Japanese)

Received October 18, 2021; Revised February 4, 2022; Accepted February 16, 2022.

Released online in J-STAGE as advance publication February 24, 2022.

*Address correspondence to:

Jun $\mathrm{Lu}$, Medical Examination Center, National Center for Global Health and Medicine, Toyama 1-21-1, Shinjuku-ku, Tokyo 162-8655, Japan.

E-mail: j-lu@hosp.ncgm.go.jp 